\title{
Stability of Second Order Equation
}

\author{
P. Shekhar ${ }^{1}$,V. Dharmaiah ${ }^{2}$ and G.Mahadevi ${ }^{3}$ \\ ${ }^{1}$ Department of Mathematics, Malla Reddy College of Engineering, Hyderabad-500014, India. \\ ${ }^{2}$ Department of Mathematics, Osmania University, Hyderabad-500007 India. \\ ${ }^{3}$ Department of Mathematics,St. Martins Engineering College, Hyderabad-500014, India
}

Abstract: In this paper we discussed the stability of the null solution of the second order differential equation . Under some unusual assumptions we obtain new stability results for this classical equation.

\section{Introduction}

Consider the second order ODE

$$
x^{\prime \prime}+2 f(t) x^{\prime}+\beta(t) x+g(t) x^{2}=0, t \in R^{+}
$$

where $R^{+}=[0, \infty), f, \beta, g: R^{+} \rightarrow R^{+}$are three given continuous functions.

The most familiar interpretation of this equation is that it describes nonlinear oscillations. Stability problems for this ODE have been studied intensively so far (see, e.g.,[13]-[15], [16]-[18], and the references therein). Recently, T.A. Burton and T. Furumochi [5] have introduced a new method to study the stability of the null solution $x=x^{\prime}=0$ of equation (1.1), which is based on the Schauder fixed point theorem. They discussed a particular case of (1.1) (one of their assumptions is $\beta(t)=1$ ) to illustrate their technique. In [8] Marosamu and Vladimirescu have proved stability results for the null solution of the same equation by using relatively classical arguments. Here, we reconsider Eq. (1.1) under more general assumptions, which require more sophisticated arguments, and prove stability results (see Theorem 2.1 below). In particular, we obtain the generalized exponential asymptotic stability of the trivial solution. See [20, p. 158] for the definition of this concept.

\section{The main result}

The following hypotheses will be required:

(i) $\quad f \in C^{\prime}\left(R^{+}\right)$and $f(t) \geq 0$ for all $t \geq 0$

(ii) $\quad \int_{0}^{\infty} f(t) d t=\infty$

(iii) there exist two constants $h, K \geq 0$ such that

$$
\left|f^{\prime}(t)+f^{2}(t)\right| \leq K f(t), t \in[h, \infty) \text {; }
$$

$$
\begin{aligned}
& \beta \in C^{\prime}\left(\mathbb{R}^{+}\right), \quad \beta \text { decreasing, and } \\
& \beta(t) \geq \beta_{0}>K^{2}, \text { for all } t \in R^{+}
\end{aligned}
$$

where $\beta_{0}$ is constant.

(v) $\quad g$ is locally Lipschitzian in $x$

These assumptions are inspired by those in [5], but are more general.

Notice that (i) and (iii) imply that $f$ is uniformly bounded (see [8], Remark 2.2).

The main result of this paper is the following theorem:

Theorem 2.1. If the assumptions (i), (iii) - (v) are fulfilled, then the null solution of (1.1) is uniformly stable. If in addition (ii) holds, then the null solution of (1.1) is asymptotically stable

Remark 2.1. Under the assumptions (i) - (v), we cannot expect to have uniform asymptotic stability for the null solution. Indeed, even in the case $g=0$ and $\beta=$ constant say $\beta(t)=1 \forall t \in R^{+}$one can construct a fundamental matrix $X(t)$ for the corresponding first order linear differential system in $\left(x, y=x^{\prime}\right)$ for which

$$
\left\|X(t) X(\tau)^{-1}\right\|
$$

does not converge to zero as $t-\tau \rightarrow \infty$ (here $\|$.$\| denotes a matrix norm)$

\section{Proof of Theorem 2.1}

we write equation (1.1) to a system

$$
\begin{gathered}
x^{\prime}=y-f(t) x \\
y^{\prime}=\left(f^{\prime}(t)+f^{2}(t)-1\right) x-f(t) y-g(t) x^{2}
\end{gathered}
$$

and write it as

$$
z^{\prime}=\left(\begin{array}{cc}
-f(t) & 1 \\
-\beta(t) & -f(t)
\end{array}\right) X+\left(\begin{array}{cc}
0 & 0 \\
f^{\prime}(t)+f^{2}(t) & 0
\end{array}\right) X+\left(\begin{array}{c}
0 \\
-g(t) x^{3}
\end{array}\right)
$$


where

$$
z^{\prime}=A(t) z+B(t) z+F(t, z)
$$

$$
\begin{gathered}
z=\left(\begin{array}{l}
x \\
y
\end{array}\right), A(t)=\left(\begin{array}{cc}
-f(t) & 1 \\
-\beta(t) & -f(t)
\end{array}\right), \mathbf{B}(t)=\left(\begin{array}{cc}
f^{\prime}(t)+f^{2}(t) & 0
\end{array}\right) \\
F(t, z)=\left(\begin{array}{c}
0 \\
-g(t) x^{2}
\end{array}\right)
\end{gathered}
$$

It is easily seen that our stability question reduces to the stability of the null solution $z(t)=0$ of system (2.3).

Let $t_{0} \geq 0$ be arbitrarily fixed and let $\boldsymbol{z}\left(\boldsymbol{t}, \boldsymbol{t}_{\mathbf{0}}\right)=\left(\begin{array}{ll}\boldsymbol{a}\left(\boldsymbol{t}, \boldsymbol{t}_{\mathbf{0}}\right) & \boldsymbol{b}\left(\boldsymbol{t}, \boldsymbol{t}_{\mathbf{0}}\right) \\ \boldsymbol{c}\left(\boldsymbol{t}, \boldsymbol{t}_{\mathbf{0}}\right) & \boldsymbol{d}\left(\boldsymbol{t}, \boldsymbol{t}_{\mathbf{0}}\right)\end{array}\right), \boldsymbol{t} \geq \mathbf{0}$ be the fundamental matrix to the linear system

$$
z^{\prime}=A(t) z
$$

which is equal to the identity matrix for $t=t_{0}$ then

$$
\begin{gathered}
a^{\prime}\left(t, t_{0}\right)=-f(t) a\left(t, t_{0}\right)+c\left(t, t_{0}\right) \\
c^{\prime}\left(t, t_{0}\right)=-\beta(t) a\left(t, t_{0}\right)-f(t) c\left(t, t_{0}\right) \\
b^{\prime}\left(t, t_{0}\right)=-f(t) b\left(t, t_{0}\right)+d\left(t, t_{0}\right) \\
d^{\prime}\left(t, t_{0}\right)=-\beta(t) b\left(t, t_{0}\right)-f(t) d\left(t, t_{0}\right)
\end{gathered}
$$

So, since $\beta$ is decreasing (hypothesis (iv)), the first two equations of (2.5) lead us to

and hence

$$
\frac{1}{2}\left(\beta(t) a\left(t, t_{0}\right)^{2}+c\left(t, t_{0}\right)^{2}\right)^{\prime} \leq-f(t)\left(\beta(t) a\left(t, t_{0}\right)^{2}+c\left(t, t_{0}\right)^{2}\right)
$$

$$
\beta(t) a\left(t, t_{0}\right)^{2}+c\left(t, t_{0}\right)^{2} \leq \beta\left(t_{0}\right) e^{-2 \int_{t_{0}}^{t} f(u) d u}, \forall t \geq t_{0} .
$$

Similarly, from the last two equations of (2.5), we get

$$
\beta(t) b\left(t, t_{0}\right)^{2}+d\left(t, t_{0}\right)^{2} \leq e^{-2 \int_{t_{0}}^{t} f(u) d u}, \forall t \geq t_{0}
$$

Consider $z=(x, y)^{T} \in R^{2}$ the norm $\|z\|=\sqrt{\beta_{0} x^{2}+y^{2}}$

For $z_{0}=\left(x_{0}, y_{0}\right)^{T} \in R^{2}$ we obtain from (2.6),(2.7), and hypothesis (iv),

$$
\begin{aligned}
& \left\|z\left(t, t_{0}\right) z_{0}\right\|=\left\|\begin{array}{l}
a\left(t, t_{0}\right) x_{0}+b\left(t, t_{0}\right) y_{0} \\
c\left(t, t_{0}\right) x_{0}+d\left(t, t_{0}\right) y_{0}
\end{array}\right\| \\
& \leq \sqrt{x_{0}^{2}+y_{0}^{2}} \times \sqrt{\beta(t)\left[a\left(t, t_{0}\right)^{2}+d\left(t, t_{0}\right)^{2}\right]+\left[c\left(t, t_{0}\right)^{2}+d\left(t, t_{0}\right)^{2}\right.} \\
& \leq \gamma \sqrt{1+\beta\left(t_{0}\right)} e^{-\int_{t_{0}}^{t} f(u) d u}\left\|z_{0}\right\|
\end{aligned}
$$

where $\gamma=\max \left\{1, \frac{1}{\sqrt{\beta_{0}}}\right\}$ Moreover, since

$$
z\left(t, t_{0}\right) z\left(s, t_{0}\right)^{-1}\left(\begin{array}{l}
0 \\
1
\end{array}\right)=\left(\begin{array}{l}
\eta\left(t, s, t_{0}\right) \\
\mu\left(t, s, t_{0}\right)
\end{array}\right), \forall t \geq s \geq t_{0} \geq 0
$$

Satisfies system (2.4), we deduce as before

$$
\begin{aligned}
\left\|z\left(t, t_{0}\right) z\left(s, t_{0}\right)^{-1}\left(\begin{array}{l}
0 \\
1
\end{array}\right)\right\| & =\sqrt{\beta_{0} \eta\left(t, s, t_{0}\right)^{2}+\mu\left(t, s, t_{0}\right)^{2}} \\
& \leq \sqrt{\beta(t) \eta\left(t, s, t_{0}\right)^{2}+\mu\left(t, s, t_{0}\right)^{2}} \\
& \leq \sqrt{\beta(s) \eta\left(t, s, t_{0}\right)^{2}+\mu\left(t, s, t_{0}\right)^{2}} e^{-\int_{s}^{t} f(u) d u} \\
& =e^{-\int_{s}^{t} f(u) d u}, \quad t \geq s \geq t_{0} \geq 0,
\end{aligned}
$$

Let us prove the first part of Theorem 2.1. Consider $z_{0} \neq 0$ with $\left\|z_{0}\right\|$ small enough, $t_{0} \geq 0$, and let us denote by $z\left(t, t_{0} z_{0}\right)$ the unique solution of (2.3) which is equal to $z_{0}$ for $t=t_{0}$. By hypotheses (i) and (v), $z\left(t, t_{0} z_{0}\right)$ is defined on a maximal right interval, say, $\left[t_{0}, l\right)$ and satisfies the following integral equation

$$
z\left(t, t_{0}, z_{0}\right)=z\left(t, t_{0}\right) z_{0}+\int_{t_{0}}^{t} z\left(t, t_{0}\right) z\left(s, t_{0}\right)^{-1}\left[B(s) z\left(s, t_{0}, z_{0}\right)+F\left(s, z\left(t, t_{0}, z_{0}\right)\right)\right] d s
$$

for all $t \in\left[t_{0}, l\right)$. From (2.8)-(2.10) we infer that

$$
\begin{aligned}
\left\|z\left(t, t_{0}, z_{0}\right)\right\| & \leq \gamma \sqrt{1+\beta\left(t_{0}\right)}\left\|z_{0}\right\| e^{-\int_{t_{0}}^{t} f(s) d u} \\
& +\int_{t_{0}}^{t} e^{-\int_{s}^{t} f(u) d u}\left[\left|f^{\prime}(s)+f^{2}(s)\right|\left|x\left(s, t_{0}, z_{0}\right)\right|+g(s) x^{2}(s)\right] d s, \quad \forall t \in\left[t_{0}, l\right)
\end{aligned}
$$

we infer from (2.11) that 


$$
\left\|z\left(t, t_{0}, z_{0}\right)\right\| \leq \gamma \sqrt{1+\beta\left(t_{0}\right)}\left\|z_{0}\right\|+D \int_{t_{0}}^{t}\left\|z\left(s, t_{0}, z_{0}\right)\right\| d s, \forall t \in\left[t_{0}, l\right)
$$

with a positive constant $\mathrm{D}$ and

$$
\left\|z\left(t, t_{0}, z_{0}\right)\right\| \leq \gamma \sqrt{1+\beta\left(t_{0}\right)} e^{D h}\left\|z_{0}\right\|, \forall t \in\left[t_{0}, l\right)
$$

Thus $z\left(t, t_{0}, z_{0}\right)$ as well as $z^{\prime}\left(t, t_{0}, z_{0}\right)$ are bounded on $\left[t_{0}, l\right)$ and so $z\left(t, t_{0}, z_{0}\right)$ can be extended to the right of $l$. This fact contradicts the maximality of $l$. Therefore $z\left(t, t_{0}, z_{0}\right)$ exists on $\left[t_{0}, l\right)$ with $l>h$.

Now, we assume $h<l<\infty$. We are going to find an estimate for $z\left(t, t_{0}, z_{0}\right)$ on the interval $[h, l)$. This time, our hypothesis (iii) comes into play. We have for all $t \in[h, l)$

$$
\begin{aligned}
\left\|z\left(t, t_{0}, z_{0}\right)\right\| & \leq \gamma \sqrt{1+\beta(h)}\left\|z\left(h, t_{0}, z_{0}\right)\right\| e^{-\int_{h}^{t} f(u) d u}+\int_{h}^{t} e^{-\int_{s}^{t} f(u) d u} K f(s)\left|x\left(s, t_{0}, z_{0}\right)\right| d s \\
& +\int_{h}^{t} e^{-\int_{s}^{t} f(u) d u} g(s) x^{2}\left(s, t_{0}, z_{0}\right) d s
\end{aligned}
$$

From (2.14) it follows that

$$
\begin{aligned}
\left\|z\left(t, t_{0}, z_{0}\right)\right\| & \leq \gamma \sqrt{1+\beta(h)}\left\|z\left(h, t_{0}, z_{0}\right)\right\| e^{-\int_{h}^{t} f(u) d u}+\int_{h}^{t} e^{-\int_{s}^{t} f(u) d u}\left|f^{\prime}(s)+f^{2}(s)\right|\left|x\left(s, t_{0}, z_{0}\right)\right| d s \\
& +\int_{h}^{t} e^{-\int_{s}^{t} f(u) d u}\left|g(s) x^{2}\left(s, t_{0}, z_{0}\right)\right| d s \\
& \leq \gamma \sqrt{1+\beta(h)}\left\|z\left(h, t_{0}, z_{0}\right)\right\| e^{-\int_{h}^{t} f(u) d u} \\
& +\int_{h}^{t} e^{-\int_{s}^{t} f(u) d u}\left[K f(s)\left|x\left(s, t_{0}, z_{0}\right)\right| d s+M f(s)\left|x\left(s, t_{0}, z_{0}\right)\right|^{3}\right] d s \\
& =\vartheta(t), \forall t \in[h, l)
\end{aligned}
$$

Then by (2.15)

$$
\begin{gathered}
\vartheta^{\prime}(t) \leq\left[\frac{K+M \vartheta(t)}{\sqrt{\beta_{0}}}-1\right] \vartheta(t) f(t) \quad \text { where } M \in\left(0, \sqrt{\beta_{0}}-K\right) \\
\vartheta(h)=\gamma \sqrt{1+\beta(h)}\left\|z\left(h, t_{0}, z_{0}\right)\right\| \\
\left\|z\left(t, t_{0}, z_{0}\right)\right\| \leq \boldsymbol{v}(t) \leq \vartheta(h) e^{\left(\frac{K+M \vartheta(h)}{\sqrt{\beta_{0}}}-1\right) \int_{h}^{t} f(u) d u}, \forall t \in[h, l]
\end{gathered}
$$

From (2.16) we can see that $z\left(t, t_{0}, z_{0}\right)$ is bounded. Since $z^{\prime}\left(t, t_{0}, z_{0}\right)$ is also bounded, it follows that $l=\infty$. Now, for $\varepsilon>0$ we denote

$$
\boldsymbol{\delta}=\boldsymbol{\delta}(\varepsilon)=\frac{\varepsilon e^{-D h}}{\gamma^{2} \sqrt{(\mathbf{1}+\boldsymbol{\beta}(\mathbf{0})(\mathbf{1}+\boldsymbol{\beta}(\boldsymbol{h})}}
$$

From (2.13) it follows that $\left\|z\left(t, t_{0}, z_{0}\right)\right\| \leq \frac{\varepsilon}{\gamma \sqrt{1+\beta(h)}}$ for all $t \in\left[t_{0}, h\right]$ provided that

$\left\|z_{0}\right\|<\delta$. Therefore, by $M \in\left(0, \sqrt{\beta_{0}}-K\right)$ and (2.16), $\left\|z\left(t, t_{0}, z_{0}\right)\right\| \leq \vartheta(h)<\varepsilon$ for all $t \geq h$. Summarizing, if $0 \leq t_{0} \leq h$, the solution $z\left(t, t_{0}, z_{0}\right)$ starting from any point, $z_{0}$, with $\left\|z_{0}\right\|<\delta$ exists on $\left[t_{0}, \infty\right)$ and satisfies $\left\|z\left(t, t_{0}, z_{0}\right)\right\|<\varepsilon$ for all $t \geq t_{0}$.

If $t_{0} \geq h$, then analogously we obtain that $l=\infty$ and

$$
\left\|z\left(t, t_{0}, z_{0}\right)\right\| \leq \vartheta \sqrt{1+\beta\left(t_{0}\right)}\left\|z_{0}\right\| e^{\left(\frac{K+M \vartheta(h)}{\sqrt{\beta_{0}}}-1\right) \int_{t_{0}}^{t} f(u) d u}, \forall t \in[h, \infty)
$$

Therefore, with the same. as before, $\left\|z_{0}\right\|<\delta$ implies again that $\left\|z\left(t, t_{0}, z_{0}\right)\right\|<\varepsilon$ for all $t \geq t_{0}$ Hence the null solution is uniformly stable. If in addition (ii) is fulfilled, then by (2.16), and (2.17) it follows that the null solution to (1.1) is asymptotically stable. The proof of Theorem 2.1 is complete.

Remark 2.2 If $f$ satisfies (i) - (iii), then $f(t)>0$ for all $t \geq h$. Let us assume, by contradiction, that $f\left(t_{1}\right)=$ 0 for some $t_{1} \geq h$. Then, one can prove that $f(t)=0$ for $t \geq t_{1}$ Indeed, if $f\left(t_{2}\right)>0$ for some $t_{2}>t_{1}$ then the function $u=\frac{1}{f}$ is well defined on the maximal interval containing $t_{2}$ on which $f>0$, say $(c, d)$, and satisfies the inequality

$$
u^{\prime}(t)+K u(t)-1 \geq 0, t \in(c, d) .
$$

This implies that

$$
\frac{d}{d t}\left(e^{K t}\left[u(t)-\frac{1}{K}\right]\right) \geq 0, t \in\left(c, t_{2}\right]
$$

i.e., the function $t \rightarrow e^{K t}\left[u(t)-\frac{1}{K}\right]$ decreasing on $\left(c, t_{2}\right]$, but this is impossible since the limit of this function is $\infty$ as $t \rightarrow c^{+}$. Thus, $f(t)=0$ for all $t \geq t_{1}$, which contradicts (ii). Therefore, we have proved that indeed $f(t)>0$ for all $t \geq h$ Consequently, the function $p(t)=\int_{0}^{\infty} f(s) d s$ is strictly increasing, at least for $t \geq h$. By the above proof we have that the null solution is generalized exponentially asymptotically stable with this $p(t)$. 
Remark 2.3. if, $\beta(t)=1, t \in R^{+}$the fundamental matrix $\mathrm{Z}\left(t, z_{0}\right)$ can be determined explicitly

$$
z\left(t, z_{0}\right)=e^{-\int_{t_{0}}^{t} f(u) d u}\left(\begin{array}{cc}
\cos \left(t-t_{0}\right) & \sin \left(t-t_{0}\right. \\
-\sin \left(t-t_{0}\right. & \cos \left(i t-t_{0}\right.
\end{array}\right)
$$

In general, this is not possible, so in our proof we had to get estimates without having an explicit form of $z\left(t, t_{0}\right)$

\section{Conclusion :}

These stability results can be extended to the case when the differential equation is vectorial equation. More precisely, assume that $f$ and $\beta$ are scalar functions satisfying the

\section{References:}

[1] Bellman.R.,Staility Theory of Differential Equations,McGraw-Hill,New York

[2] Burton T.A, and Furumochi,Tetsuo (2001) Fixed points and prolem in stability Theory for ordinary and functional Differential quations, Dynamical System. April 10, 89-116

[3] Burton T.A, and Furumochi,Tetsuo (2002) Asymptotic behaviour of solution of functional Differential Equations, by fixed point theorem Dynamical system 11,499-519.

[4] Burton T.A, and Furumochi,Testuo (2002) Krasnoselskiis fixed point theorem and stability of nonlinear Analysis 49, $445-454$.

[5] Burton T.A, and Furumochi,Testuo (2005) Asymptotic behaviour of non linear functional Differential Equations, by Schauder's theorem Nonlinear Stud. 12,73-84.

[6] Coddington, E.A. and Levinson, Theory of Differential Equations,McGraw-Hill,New York.

[7] C. Corduneanu, Principles of Differential and Integral Equations, Allyn and Bacon, Boston, 1971.

[8] Gh. Moro sanu, C. Vladimirescu, Stability for a nonlinear second order ODE, CEU Preprint, August 2003, to appear in Funkcialaj Ekvacioj.

[9] Hale, Jack K., Ordinary Differential Equations, Wiley, New York, 1969.

[10] Hartman, Philip, Ordinary Differential Equations, Wiley, New York, 1964.

[11] Hatvani, L., Integral conditions on the asymptotic stability for the damped oscillator with small damping, Proc. Amer. Math. Soc. 124(1996), 415-422.

[12] L.C. Piccinini, G. Stampacchia, G. Vidossich, Ordinary Differential Equations in IRn. Problems and Methods, Applied Mathematical Sciences, 39, New York, Springer-Verlag, 1984.

[13] L. Hatvani, On the stability of the zero solution of certain second order non-linear differential equations, Acta Scientiarum Mathematicarum,32, 1-9 (1971).

[14] L. Hatvani, A generalization of the Barbashin-Krasovskij theorems to the partial stability in nonautonomous systems, Colloquia Mathematica Societatis Janos Bolyai, Vol. 30: Qualitative Theory of Differential Equations, Szeged, Hungary, 1979, pp. 381-409.

[15] L. Hatvani, Integral conditions on the asymptotic stability for the damped linear oscillator with small damping, Proceedings of the American Mathematical Society, 124, 415-422 (1996).

[16] P. Pucci, J. Serrin, Precise damping conditions for global asymptotic stability for nonlinear second order systems, Acta Mathematica, 170,275-307 (1993).

[17] P. Pucci, J. Serrin, Asymptotic stability for intermittently controlled nonlinear oscillators, SIAM Journal on Mathematical Analysis, 25, 815-835 (1994).

[18] P. Pucci, J. Serrin, Precise damping conditions for global asymptoticstability for non-linear second order systems, II, Journal of Differential Equations, 113, 505-534 (1994).

[19] Smart, D. R., Fixed Point Theorems, Cambridge Univ. Press, Cambridge, 1974.

[20] V. Lakshmikantham, S. Leela, Differential and Integral Inequalities.Theory and Applications, Vol. I: Ordinary Differential Equations, Academic Press, New York and London, 1969. 